\title{
Evaluating the utility of a point-of-care glucometer for the diagnosis of gestational diabetes
}

\author{
Sumaiya Adam ${ }^{1, *}$ and Paul Rheeder ${ }^{2}$ \\ ${ }^{1}$ Department of Obstetrics and Gynaecology, University of Pretoria, Pretoria, South Africa \\ ${ }^{2}$ Department of Internal Medicine, University of Pretoria, Pretoria, South Africa \\ * Correspondence: Sumaiya Adam, Private Bag X169, Pretoria 0001, South Africa. \\ Email: sumaiya.adam@up.ac.za
}

\begin{abstract}
Objective: To investigate the performance of the Roche Accuchek Active glucometer in diagnosing gestational diabetes mellitus (GDM) versus the gold-standard laboratory test.

Methods: In a prospective cohort observational study at a primary healthcare clinic in Johannesburg, South Africa, pregnant women, excluding known diabetics, were recruited between 2013 and 2016. A 75-g 2-hour oral glucose tolerance test (OGTT) was scheduled at 24-28 gestational weeks. Glucose was measured in venous blood (laboratory) and capillary blood (glucometer). GDM was diagnosed via FIGO criteria. Diagnostic accuracy was evaluated by calculating the sensitivity, specificity, and coefficient of variance (CV) of the glucometer test, and by Bland-Altman plots.
\end{abstract}

Results: Data from 529 women were analyzed. Of these, 141 (26.7\%) and 79 (14.9\%) were diagnosed with GDM by laboratory and glucometer measurements, respectively. The CV of the glucometer ranged from $15 \%$ to $17 \%$. Bland-Altman plots showed a positive bias of the glucometer results at 0 hours, but a negative bias at 1 and 2 hours of the OGTT. The sensitivity and specificity of the glucometer for the diagnosis of GDM were $27.0 \%$ and $89.4 \%$, respectively.

Conclusion: Use of the Roche Accuchek Active glucometer for the diagnosis of GDM cannot be recommended.

Keywords: Accuracy; Gestational diabetes; Glucometer; Point-of-care testing

\section{INTRODUCTION}

After decades of research, there is now almost universal consensus regarding the screening and diagnostic criteria for gestational diabetes mellitus (GDM).[1] Hyperglycemia in pregnancy is associated with adverse perinatal outcomes and increases long-term risks for both mother and child.[2-4] Despite investigations into other screening and diagnostic tests, 
the 75-g 2-hour oral glucose tolerance test (OGTT) remains the cornerstone of diagnosis.[5] Furthermore, there is a shift away from conducting traditional risk-factor-based screening among high-risk women only to universal screening for GDM among all pregnant women.[1]

The OGTT is not without limitations. Both a trained phlebotomist and laboratory facilities for glucose measurement are required. In addition, the results of the OGTT are available only several days later, potentially necessitating more clinic visits by the woman. With over a million pregnancies registered in South Africa every year, this places an enormous burden on the healthcare system and the pregnant women.[6]

To make universal screening for GDM feasible, a point-of-care (POC) test for glucose with good accuracy and precision is required, especially in low-resource settings.[1] It is generally accepted in clinical practice that capillary blood glucose and venous plasma glucose measurements are comparable.[7-9] However, laboratory tests are performed on venous plasma, whereas POC tests are usually performed on capillary whole blood. Glucose levels vary depending on the source of the blood sample used for analysis; this variation is attributed to differences in glucose extraction by tissues, perfusion, oxygenation, $\mathrm{pH}$, and temperature.[10] The increased volume of distribution associated with pregnancy may further affect these measurements.[11] Capillary blood glucose concentrations have been shown to be significantly higher than venous glucose concentrations.[10]

Despite advances in glucometer technology over the past decade, the glucose POC device does not perform consistently on statistical analysis.[12,13] Most studies have focused on evaluating use of the glucometer for monitoring and guiding insulin management for known patients with diabetes mellitus, $[12,13]$ and few have investigated use of the POC glucometer for the diagnosis of GDM.[14-16] The POC glucometer represents an attractive option for the diagnosis of GDM, especially in low-resource settings where laboratory services and transportation may not be readily available. Furthermore, a POC device would facilitate timely diagnosis of GDM and initiation of its management. In turn, this might improve adherence to screening guidelines, especially if universal screening for GDM is implemented. A POC glucometer would also facilitate a diagnosis of GDM based on elevated fasting glucose levels alone,[17] which is important for populations where most cases of GDM are currently diagnosed on the basis of an elevated fasting plasma glucose alone, including the present study population.

The use of glucometers for monitoring and management of diabetes mellitus has been extensively studied and is generally accepted as part of care of the diabetic patient, despite variations in the performance of POC devices relative to the gold-standard laboratory test.[12-20] Although FIGO guidelines recommend use of the glucometer for the diagnosis of GDM in low-resource settings,[1] there is less robust, and often conflicting, evidence regarding their use for GDM diagnosis.[14-16] The aim of the present study was therefore to investigate the performance of the Roche Accuchek Active glucometer, which is the most commonly available POC device in the study setting, in the diagnosis of GDM. 


\section{MATERIALS AND METHODS}

The present analysis formed part of a larger study of screening strategies for GDM in South Africa. In a prospective cohort observational study, 1000 pregnant women were recruited at a primary healthcare clinic in Johannesburg, South Africa, between September 1, 2013, and June 30, 2016. Approval for the study was obtained from the University of Pretoria, Faculty of Health Sciences Ethics Committee (Protocol 180/2012). Informed consent was obtained from every woman prior to enrollment in the study.

The sample size was calculated by using a $5 \%$ margin of error and a $95 \%$ confidence interval $(\mathrm{Cl})$, which determined that 400 women would be needed to complete the study. Considering loss to follow-up (50\%), pregnancy loss (15\%), and patient migration (20\%), a sample size of 1000 (to the nearest 100) was calculated.

Women at less than 26 gestational weeks were recruited. Those known to have diabetes mellitus or were more than 26 weeks pregnant were excluded.

At recruitment, each woman completed a questionnaire of demographic data and underwent an evaluation of risk factors for GDM. Gestational age was determined by the woman's last normal menstrual period, ultrasound scan, or measurement of fundal height. Random blood glucose was measured at recruitment on both a POC device and the laboratory. If the random glucose level was greater than $11.1 \mathrm{mmol} / \mathrm{L}(199.8 \mathrm{mg} / \mathrm{dL})$, the woman was referred to the local hospital for further management of overt diabetes. Otherwise, a 75-g 2-hour OGTT was scheduled at 24-28 gestational weeks. GDM was diagnosed on the basis of FIGO criteria: namely, any one glucose value corresponding to $5.1 \mathrm{mmol} / \mathrm{L}(91.8 \mathrm{mg} / \mathrm{dL})$ or higher at $0 \mathrm{~h}, 10 \mathrm{mmol} / \mathrm{L}(180 \mathrm{mg} / \mathrm{dL})$ or higher at 1 hour, or $8.5 \mathrm{mmol} / \mathrm{L}(153 \mathrm{mg} / \mathrm{dL})$ or higher at 2 hour.[1]

Venous blood was drawn by a trained research nurse into a fluoridated tube and was stored on ice until delivery to the laboratory as soon as possible, simulating the real clinical situation. The sample was centrifuged on arrival at the laboratory, unlike other studies in which it was centrifuged within 5-30 minutes.[21] The laboratory is accredited by the South African National Accreditation System, and uses the Beckman DXc hexokinase method to measure glucose. The laboratory test had a mean imprecision of $1.68,1.38$, and 1.48 at glucose levels of $2.4 \mathrm{mmol} / \mathrm{L}(43.2 \mathrm{mg} / \mathrm{dL}), 12 \mathrm{mmol} / \mathrm{L}(216 \mathrm{mg} / \mathrm{dL})$, and $22 \mathrm{mmol} / \mathrm{L}$ $(396 \mathrm{mg} / \mathrm{dL})$, respectively. The mean bias was $3.65,1.36$, and 1.27 at glucose levels of $2 \mathrm{mmol} / \mathrm{L}(36 \mathrm{mg} / \mathrm{dL}), 7 \mathrm{mmol} / \mathrm{L}(126 \mathrm{mg} / \mathrm{dL})$, and $15 \mathrm{mmol} / \mathrm{L}(270 \mathrm{mg} / \mathrm{dL})$, respectively.

At the same visit, capillary glucose was tested on the Roche Accuchek Active POC device (Roche Diagnostics, Mannheim, Germany). Two trained research nurses performed the POC glucose measurements. The woman's hands were cleaned prior to obtaining the capillary sample. The test was carried out within 5 minutes of venepuncture.

The Roche Accuchek Active meter measures glucose by reflective photometry using the hexokinase method. The glucose values displayed correspond to the estimated plasma glucose concentration even though the device measures glucose in whole blood. The POC device was calibrated in accordance with the manufacturer's guidelines and test strips were 
stored appropriately. During the study, four different lot numbers were used so that all test strips remained within their expiration date; however differences in test strips used for glucometers might diminish analytical quality because studies have shown that significant variability exists between test strips.[22, 23]

The study data were analyzed by using Stata version 13 (StataCorp, College Station, TX USA). Continuous variables were analyzed by Student $t$ test. Glucose measurements taken at 0 hours, 1 hour, and 2 hours were analyzed separately. Laboratory glucose measurements were regarded as the "gold standard".

Capillary glucometer results were evaluated in accordance with ISO 15197:2013

guidelines.[24] These recommend that, for a blood glucose level of $4.2 \mathrm{mmol} / \mathrm{L}(75.6 \mathrm{mg} / \mathrm{dL})$ or less on laboratory testing, glucometer results should be within $0.83 \mathrm{mmol} / \mathrm{L}$

(14.94 mg/dL) for at least $99 \%$ of samples tested. For blood glucose levels above $4.2 \mathrm{mmol} / \mathrm{L}$ $(75.6 \mathrm{mg} / \mathrm{dL}$ ) on laboratory testing, glucometer results should be within $15 \%$ for at least $99 \%$ of samples tested.[24]

Multiple statistical methods were used to analyze the accuracy of the glucometer. BlandAltman plots were generated for glucose measurements at 0 hours, 1 hour, and 2 hour to assess agreement between the glucometer and laboratory assays. Acceptable limits of agreement were defined as -0.5 to +0.5 . The coefficient of variation (CV), defined as the ratio of the SD to the mean, was determined to assess variability, and a CV of less than $5 \%$ was taken as acceptable. The Youden index (J) was used to evaluate the performance of the glucometer test, and was calculated by the formula J=sensitivity + specificity -1 . Receiver operator characteristic (ROC) curve analysis was used to determine the sensitivity and specificity of the glucometer for the diagnosis of GDM in clinical practice. A P value of less than 0.05 was considered to be statistically significant.

\section{RESULTS}

Of the 1000 pregnant women recruited, 82 (8.2\%) experienced fetal loss and did not complete the study, $163(16.3 \%)$ moved from the area, 194 (19.4\%) were lost to follow-up, and $7(0.7 \%)$ withdrew consent. In addition, the paired glucose data were incomplete for 25 (2.5\%) women. Thus, 529 (52.9\%) women had complete data and formed the study population (Table 1).

Table 1. Clinical characteristics of the study population $(n=529)$

\section{Characteristic}

Age, y

Gestational age at recruitment, wk

BMI

Hemoglobin, g/dL (mmol/L)

Glycated hemoglobin/HbA1c, \% (mmol/mol) 5.2 (33)
Mean value

27.3

18.7

26.5

$12.3(7.7)$
$95 \% \mathrm{Cl}$

$26.8-27.8$

18.2-19.1

26.1-27.0

$12.2-12.5(7.5-7.8)$

$5.2-5.2(33.0-34.0) \quad 3.8-6.5$

Abbreviations: BMI, body mass index (calculated as weight in kilograms divided by the square of height in meters); $\mathrm{Cl}$, confidence interval. 
Gestational age was determined by last normal menstrual period for $443 / 529$ (83.7\%) women and by early ultrasound for $86 / 529$ (16.3\%) women. A hemoglobin level of less than $11 \mathrm{~g} / \mathrm{dL}(6.8 \mathrm{mmol} / \mathrm{L})$ was considered to indicate anemia. The presence of anemia had no significant effect on the glucometer measurement $(P=0.903$ at 0 hours, $P=0.331$ at 1 hour, and $P=0.045$ at 2 hours), but had an effect on the laboratory measurement of glucose ( $P=0.006$ at 0 hours, $P=0.162$ at 1 hour, and 0.068 at 2 hours).

Among the 529 women, 141 (26.7\%) women were diagnosed with GDM via the goldstandard laboratory measurement. By contrast, 79 (14.9\%) women were diagnosed with GDM via glucometer measurement. The mean glucose levels at 0 hours, 1 hour and 2 hours of the OGTT measured by the laboratory and the glucometer are shown in Table 2.

Table 2. Mean glucose levels in OGTT

\begin{tabular}{|c|c|c|c|c|c|}
\hline \multirow{2}{*}{ Test } & \multicolumn{2}{|c|}{ Glucometer, capillary } & \multicolumn{2}{|c|}{ Laboratory, venous } & \multirow{2}{*}{$P$ value } \\
\hline & $\mathrm{mmol} / \mathrm{L}$ & $\mathrm{mg} / \mathrm{dL}$ & $\mathrm{mmol} / \mathrm{L}$ & $\mathrm{mg} / \mathrm{dL}$ & \\
\hline \multicolumn{6}{|l|}{$\mathrm{Oh}$} \\
\hline Mean $(95 \% \mathrm{Cl})$ & $4.4(4.3-4.5)$ & $79.0(78.1-80.1)$ & $4.8(4.7-4.8)$ & 85.5 (83.9-86.9) & 0.162 \\
\hline Range & $2.7-8.4$ & $48.6-151.2$ & $2.1-13.4$ & $37.8-241.2$ & \\
\hline \multicolumn{6}{|l|}{$1 \mathrm{~h}$} \\
\hline Mean $(95 \% \mathrm{Cl})$ & $6.6(6.4-6.7)$ & $118.1(115.9-120.2)$ & $5.9(5.8-6.0)$ & $105.8(103.5-108.2)$ & $<0.001$ \\
\hline Range & $2.6-12.9$ & $46.8-232.2$ & $2.7-12.1$ & $48.6-217.8$ & \\
\hline \multicolumn{6}{|l|}{$2 \mathrm{~h}$} \\
\hline Mean $(95 \% \mathrm{Cl})$ & $6.0(5.9-6.1)$ & 107.8 (106.0-109.6) & $5.6(5.4-5.7)$ & $99.0(97.7-101.7)$ & $<0.001$ \\
\hline Range & $3.3-15.5$ & $59.4-279.0$ & $2.8-13.8$ & $50.4-248.4$ & \\
\hline
\end{tabular}

Abbreviation: OGTT, oral glucose tolerance test.

The CV of the glucometer test was 16\%, 17\%, and 15\% at 0, 1, 2 hours, respectively, indicating poor precision of the Roche Accuchek Active glucometer relative to laboratory measurements, which range from $0.97 \%$ to $3.41 \%$.

The glucometer results were evaluated in terms of the ISO guidelines (Table 3). Overall, 216 (74.5\%) of 290 glucometer readings were within $0.83 \mathrm{mmol} / \mathrm{L}(14.94 \mathrm{mg} / \mathrm{dL})$ of the corresponding laboratory measurement for glucose levels of $4.2 \mathrm{mmol} / \mathrm{L}(75.6 \mathrm{mg} / \mathrm{dL})$ or less, and 758 (58.4\%) of 1297 glucometer measurements were within $15 \%$ of the laboratory measurement for glucose levels above $4.2 \mathrm{mmol} / \mathrm{L}(75.6 \mathrm{mg} / \mathrm{dL})$.

Table 3. Stratification of glucometer readings as per ISO guidelines ${ }^{\text {.a }}$

\begin{tabular}{|c|c|c|}
\hline Test & $\begin{array}{c}\text { No. (\%) of samples } \leq 4.2 \mathrm{mmol} / \mathrm{L}(75.6 \mathrm{mg} / \mathrm{dL}) \text { within } \\
0.83 \mathrm{mmol} / \mathrm{L}(14.94 \mathrm{mg} / \mathrm{dL}) \text { of lab value }\end{array}$ & $\begin{array}{c}\text { No. of samples }>4.2 \mathrm{mmol} / \mathrm{L}(75.6 \mathrm{mg} / \mathrm{dL}) \\
\text { within } 15 \% \text { of lab value) }\end{array}$ \\
\hline $\mathrm{Oh}$ & $140 / 161(87.0)$ & $232 / 368(63.0)$ \\
\hline $1 \mathrm{~h}$ & 43/53 (28.3) & $218 / 476(45.8)$ \\
\hline $2 \mathrm{~h}$ & $33 / 76(44.4)$ & $308 / 453(68.0)$ \\
\hline Overall & $216 / 290(74.5)$ & $758 / 1297(58.4)$ \\
\hline
\end{tabular}


Bland-Altman plots were used to assess agreement between the glucometer and laboratory measurements of glucose (Fig. 1). The plot at 0 hours (Fig. 1A) showed an average glucose level (across laboratory and glucometer measurements) of 3.3-9.0 mmol/L (58.5$162.0 \mathrm{mg} / \mathrm{dL}$ ). There was an acceptable positive bias of 0.35 (95\% confidence interval $[\mathrm{Cl}]$, 0.26-0.44); in other words, the laboratory measurements were higher on average than the glucometer results. The difference between the laboratory measurement and the glucometer measurement ranged from 1.7 to $2.4 \mathrm{mmol} / \mathrm{L}(30.7-43.3 \mathrm{mg} / \mathrm{dL})$.

The Bland-Altman plot at 1 hour (Fig. 1B) showed an average glucose level of 3.411.0. $\mathrm{mmol} / \mathrm{L}(61.2-197.1 \mathrm{mg} / \mathrm{dL})$. There was an unacceptable negative bias of $-0.68(95 \%$ $\mathrm{Cl},-0.78$ to -0.59$)$; in other words, the laboratory measurements were lower on average than the glucometer results. The difference between the laboratory measurement and the glucometer measurement ranged from -3.0 to $1.6 \mathrm{mmol} / \mathrm{L}(-53.5$ to $28.8 \mathrm{mg} / \mathrm{dL}$ ).

The Bland-Altman plot at 2 hours (Fig. 1C) showed an average glucose level of 3.8$11.3 \mathrm{mmol} / \mathrm{L}(67.5-202.5 \mathrm{mg} / \mathrm{dL})$. There was an acceptable negative bias of $-0.45(95 \% \mathrm{Cl}$, -0.54 to -0.36$)$; in other words, the laboratory measurements were lower on average than the glucometer results. The difference between the laboratory measurement and the glucometer measurement ranged from -2.6 to $1.7 \mathrm{mmol} / \mathrm{L}(-47.4$ to $31.3 \mathrm{mg} / \mathrm{dL}$ ). 


\section{(A) $0 \mathrm{hr}$}

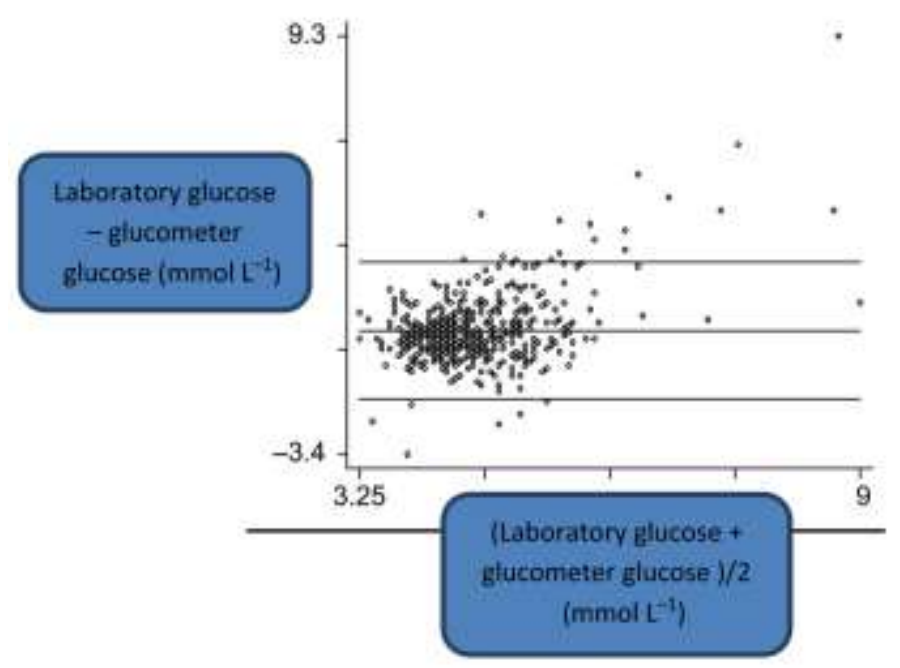

(B) $1 \mathrm{hr}$

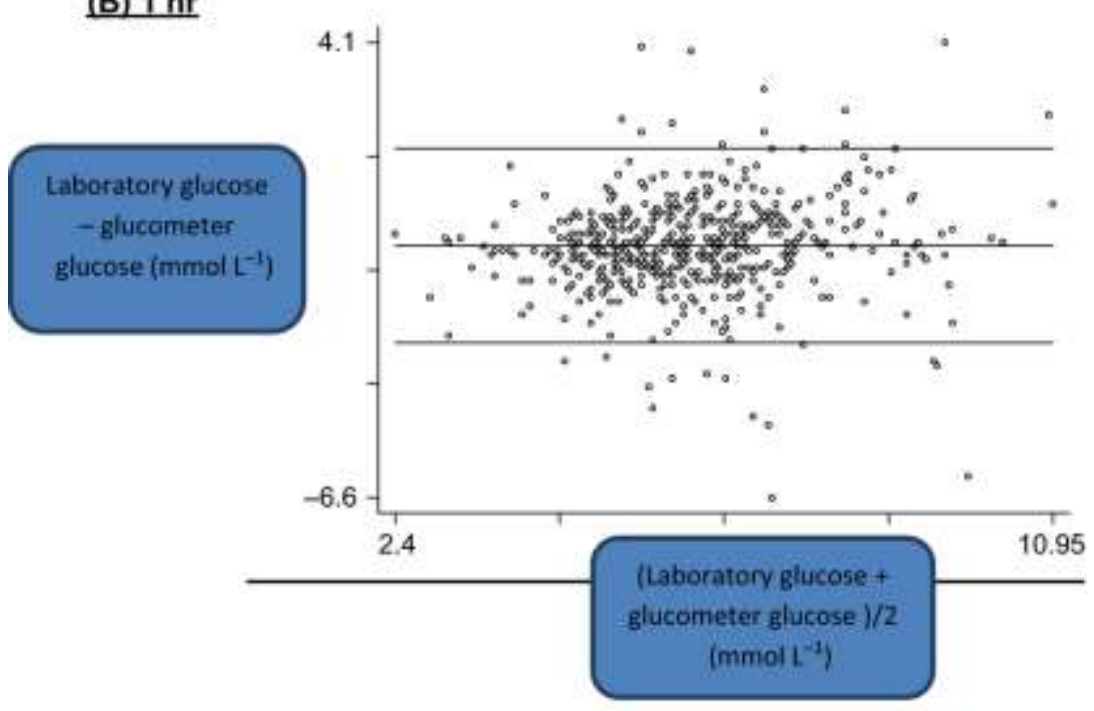

\section{(C) $2 \mathrm{hr}$}

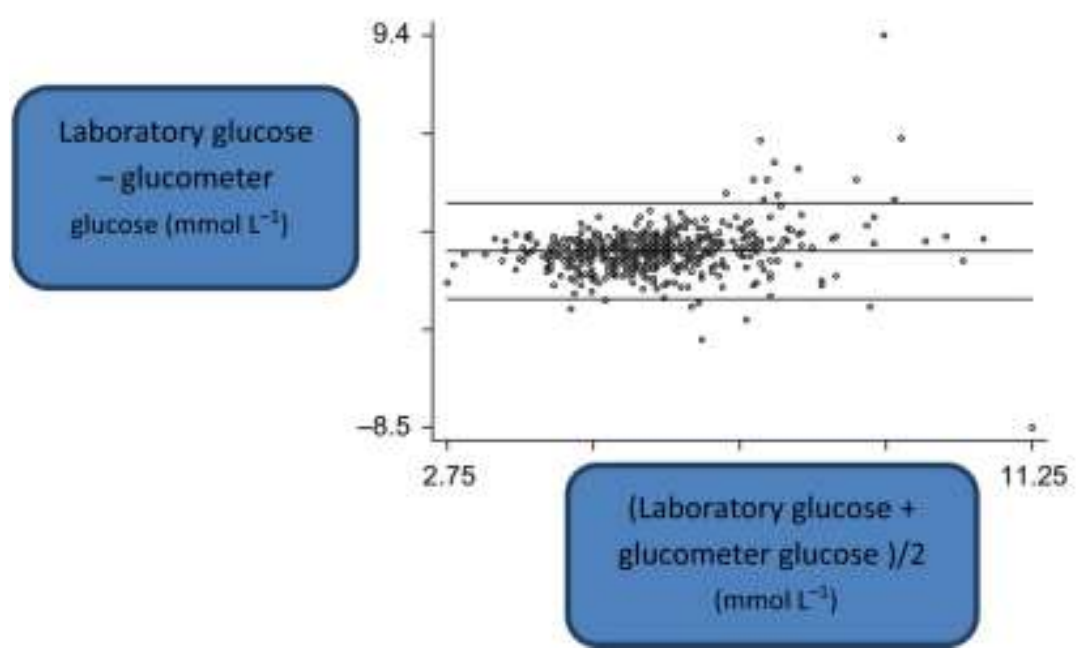

Figure 1. Bland-Altman plots of glucose measurements at 0 hours (A), 1 hour (B), and 2 hours (C) of the $75-\mathrm{g}$ oral glucose tolerance test. 
(a) $0 \mathrm{hr}$

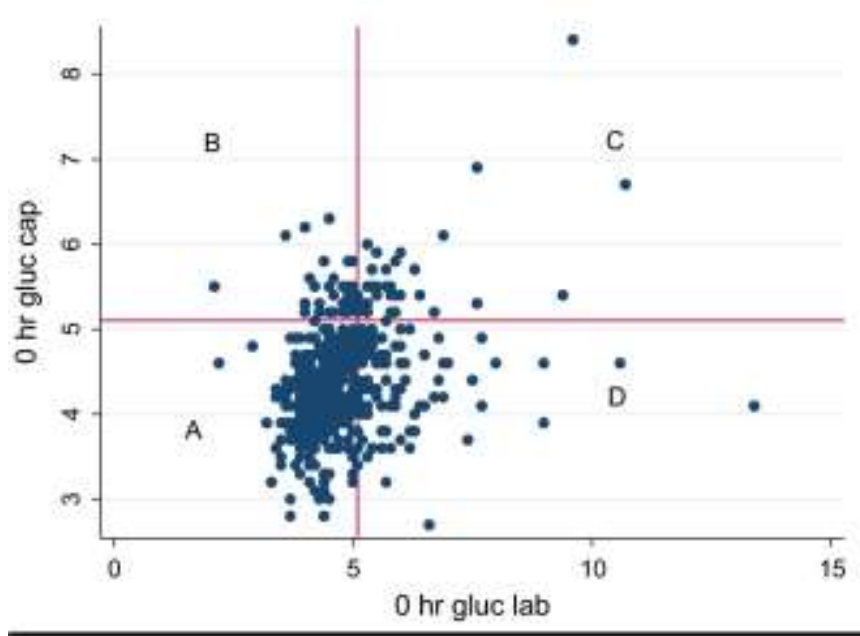

(b) $1 \mathrm{hr}$

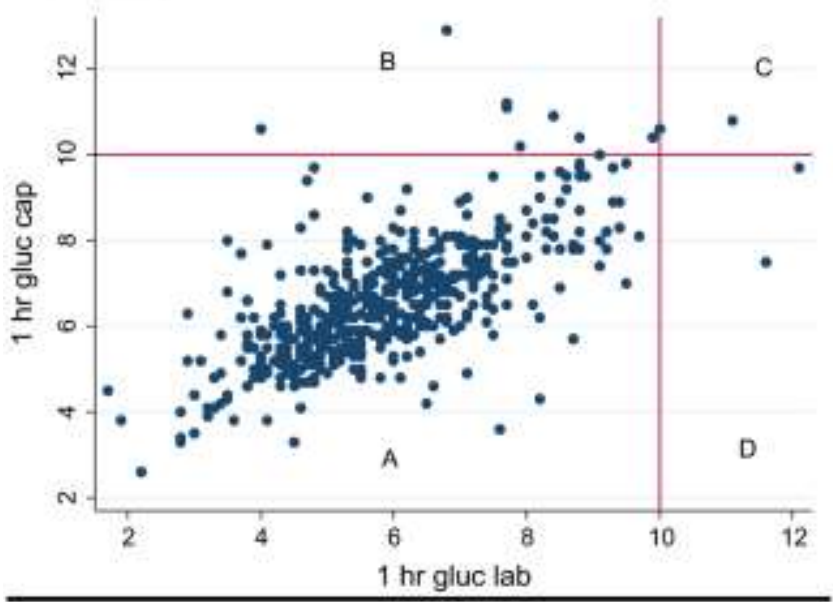

(c) $2 \mathrm{hr}$

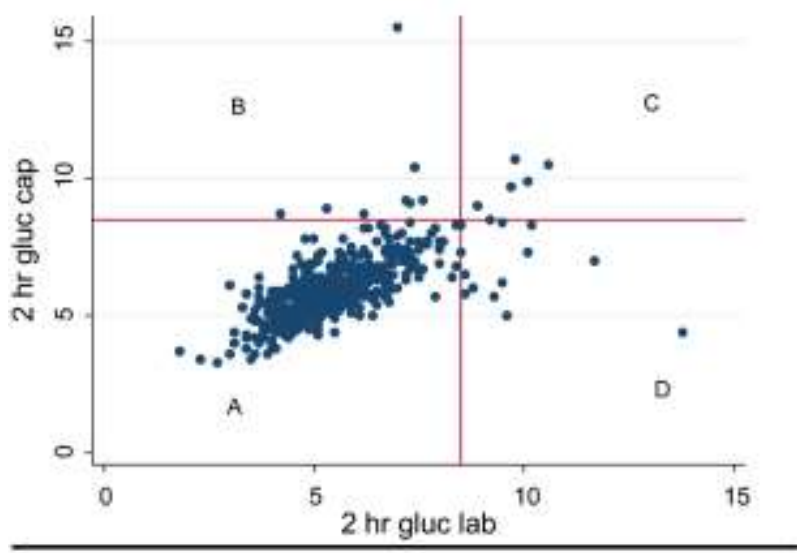

(d)

\begin{tabular}{|c|c|c|c|}
\hline Region & $\begin{array}{c}\text { 0-hr test } \\
(\mathbf{n = 5 2 9 )}\end{array}$ & $\begin{array}{c}\text { 1-hr test } \\
(\mathbf{n = 5 2 9 )}\end{array}$ & $\begin{array}{c}\text { 2-hr test } \\
(\mathbf{n = 5 2 9 )}\end{array}$ \\
\hline A & $374(70.7)$ & $517(97.7)$ & $504(95.3)$ \\
\hline B & $34(6.4)$ & $9(1.7)$ & $8(1.5)$ \\
\hline C & $31(5.9)$ & $1(0.2)$ & $5(0.9)$ \\
\hline D & $90(17)$ & $2(0.4)$ & $12(2.3)$ \\
\hline
\end{tabular}

Figure 2. Scatter plots of laboratory glucose measurements vs glucometer measurements at 0 hours (a), 1 hour (b), and 2 hours (c) of the oral glucose tolerance test. (d) Number (percentage) of women in each region. The reference lines are based on the FIGO diagnostic criteria for GDM.[1] Regions (A) and (C) show concordance between laboratory glucose and glucometer readings (i.e., both methods report measurement above or below the thresholds, and thus do not affect the diagnosis of GDM). Regions (B) and (D) demonstrate discordant sample sets that would lead to misdiagnosis of GDM. Abbreviations: GDM, gestational diabetes mellitus; gluc, glucose; cap, capillary; lab, laboratory. 
The glucometer was found to have a sensitivity of $27.0 \%$ and a specificity of $89.4 \%$ (Table 4 ). The Youden index was 0.16 and accuracy was calculated to be $72.8 \%$.

Table 4. Diagnostic characteristics of point-of-care device in the diagnosis of GDM ( $n=529)$.a

Diagnosis by lab test

GDM

No GDM

Total
GDM

$41(7.7)$

79 (14.9)
Diagnosis by glucometerb

No GDM

$103(19.5)$

347 (65.6)

$450(85.1)$
Total

$141(26.7)$

$388(73.3)$

$529(100)$

\footnotetext{
${ }^{a}$ Values are given as number (percentage).

${ }^{\mathrm{b}}$ Receiver operator characteristic curve analysis of glucometer diagnosis: area under curve, 0.58; sensitivity, $27.0 \%$; specificity, $89.4 \%$; positive predictive value, $48.1 \%$; negative predictive value, $77.1 \%$.
}

Clinical accuracy was further evaluated by scatter plots (Fig. 2). Overall, 137 of the 141 (97.2\%) women with an abnormal OGTT by laboratory test had a fasting glucose level of $5.1 \mathrm{mmol} / \mathrm{L}(91.8 \mathrm{mg} / \mathrm{dL})$. The fasting plasma glucose had a good predictive value for the diagnosis of GDM with an area under the ROC curve (AUC) of 0.99. By contrast, the capillary glucose measurement performed poorly with an AUC of 0.58 ; in other words, only 68 $(48.2 \%)$ measurements were $5.1 \mathrm{mmol} / \mathrm{L}(91.8 \mathrm{mg} / \mathrm{dL})$ or above on the glucometer.

\section{DISCUSSION}

The present study found that the glucometer performed poorly as compared with the laboratory when used for the diagnosis of GDM. It did not meet the ISO criteria and so has poor analytic accuracy.[24] It also has poor clinical accuracy, as demonstrated by the large number of women with GDM who would not have been diagnosed if their glucose level had been measured only by glucometer (Table 4).

Previous studies investigating a POC glucometer for the diagnosis of GDM have recommended its use in clinical practice.[14-16] A recent South African study found that most glucometers were acceptable, although the authors warned about the variability among different meters and the need for independent comparison.[25] The present study found that the Roche Accuchek Active glucometer performed poorly when used for the diagnosis of GDM. This is consistent with a review of POC glucometers that found that a reliable glucose reading relative to the laboratory reference is achieved only by approximately $50 \%$ of POC meters.[26]

In the present study, the glucometer was found to have poor sensitivity and specificity for the diagnosis of GDM. Overall, 103 (19.5\%) cases of GDM would not have been diagnosed by the glucometer alone. Numerous variables can affect the measured glucose level, including the POC device or test strips, patient medications, hematocrit level, blood $\mathrm{pH}$, the site from which blood was obtained, and the detection method used by the POC device.[27] Balaji et al.[19] also investigated the use of a glucometer in a low-resource setting, and reported a poor correlation between the glucometer and the laboratory. They suggested using a lower cut-off for the capillary reading; however, their proposal to use the glucometer as a screening tool would necessitate two OGTTs, which would increase the 
number of visits to the clinic and might also deter women from have the test at all owing to the adverse effects of the oral glucose load.

It was previously demonstrated that capillary glucose values are higher than venous glucose readings.[10] In the present study, however, the venous glucose measurements at 0 hours were higher. This might be due to oxidation in the sample caused by the longer time between sampling and centrifugation. Alternatively, the variation in glucometer strips might have led to altered enzyme activity or enzyme coverage, thus resulting in lower values measured on the glucometer.[28]

Most cases of GDM in the study were diagnosed on the basis of fasting glucose. We considered the possibility of applying a correction factor to the glucometer, but were unable to derive a simple user-friendly formula. Furthermore, correction factors do not perform well when applied to the general population, and thus would not be feasible in a universal screen for GDM.

The advantages of the study include its large patient numbers in a low-resource real-world setting. In addition, two research nurses were used to minimize variability (two research nurses were needed to accommodate their other commitments during the study period). The study also has limitations. First, only one POC glucometer system was tested, and four lots of test strips were used, thereby increasing variability. For the laboratory test, blood was not centrifuged within the recommended 30 minutes owing to the distance between the laboratory and the clinic. Last, citrate tubes were not used for the collection of venous blood for glucose measurements.

The use of a POC device remains an alluring tool for the diagnosis of GDM. There have been conflicting results from recent studies.[19, 20] Whereas Balaji et al.[19] found a poor correlation, Jadhav et al.[20] reported $100 \%$ correlation between the glucometer and the laboratory. It can be concluded that glucometers have variable performance and should be used cautiously for the diagnosis of GDM.

On the basis of the present study, use of the Roche Accuchek Active glucometer for the diagnosis of GDM cannot be recommended. There is a need to test and improve the accuracy and precision of POC glucometers for the diagnosis of GDM. Newer technologies, such as smartphone measurements of glucose or continuous glucose monitoring, might have to be considered as alternatives to the OGTT for the diagnosis of GDM.

\section{AUTHOR CONTRIBUTIONS}

SA contributed to the conception and development of the study, data collection, statistical analyses, and writing the manuscript. PR contributed to the conception and development of the study, statistical analyses, and writing the manuscript.

\section{ACKNOWLEDGMENTS}

The authors thank the Society for Endocrinology, Metabolism and Diabetes of South Africa, the South African Sugar Association, and Roche for financial support of the study. 


\section{CONFLICTS OF INTEREST}

The authors have no conflicts of interest.

\section{REFERENCES}

1. Mclntyre HD, Colaguiri S. Diagnosis of GDM: A suggested consensus. Best Pract Res Clin Obstet Gynaecol. 2015;29:194-205.

2. The HAPO Study Cooperative Research Group. Hyperglycaemia and adverse pregnancy outcomes. N Engl J Med. 2008;358:1991-2002.

3. International Association of Diabetes and Pregnancy Study Groups Consensus Panel. International association of diabetes and pregnancy study groups recommendations on the diagnosis and classification of hyperglycaemia in pregnancy. Diabetes Care. 2010;33:676-682.

4. Damm P, Houshmond-Oeregaard A, Kelstrup L, Lauenborg J, Mathiesen ER, Clausen TD. Gestational diabetes mellitus and long-term consequences for mother and offspring: A view from Denmark. Diabetologia. 2016;59:1396-1399.

5. Farrar D, Duley L, Lawlor DA. Different strategies for diagnosing gestational diabetes to improve maternal and infant health. Cochrane Database Syst Rev. 2015;(1):CD007122.

6. Pattinson RC, ed. Saving Mothers 2011-2013: The Sixth Report of the National Committee for Confidential Enquiries into Maternal Deaths in South Africa. Pretoria: Government Printer; 2014.

7. Kuwa K, Nakayama T, Hoshino T, Tominaga M. Relationships of glucose concentrations in capillary whole blood, venous whole blood and venous plasma. Clin Chim Acta. 2001;307:187-192.

8. Colagiuri S, Sandbaek A, Carstensen B, et al. Comparability of venous and capillary glucose measurements in blood. Diabet Med. 2003;20:953-956.

9. Sacks DB, ed. Guidelines and recommendations for laboratory analysis in the diagnosis and management of diabetes mellitus. Published 2011.

http://www.aacc.org/ /media/practice-

guidelines/diabetesmellitus/diabetesmellitusentirelmpg.pdf?la=en. Accessed June 21, 2017.

10. Hod M, Kapur A, Sacks D, et al. The International Federation of Gynecology and Obstetrics (FIGO) initiative on gestational diabetes mellitus: A pragmatic guide for diagnosis, management, and care. Int J Gynecol Obstet. 2015;131(S3):S173-S211.

11. Soma-Pillay P, Nelson-Piercy C, Tolppanen H, Mebazaa A, Mebazaa A. Physiological changes in pregnancy: Review articles. Cardiovasc J Afr. 2016;27:89-94.

12. McGrath RT, Donnelly VC, Glastras SJ, et al. Evaluation of blood glucose meter efficacy in an antenatal diabetes clinic. Diabetes Technol Ther. 2016;18:68-74.

13. Kumar V, Indukuri D, Bhat N. Analysis of five glucometers available in India. Int J Diabetes Dev Ctries. 2015;35:189-196.

14. Daly N, Carroll C, Flynn I, Harley R, Maguire PJ, Turner MJ. Evaluation of point-of-care maternal glucose measurements of the diagnosis of gestational diabetes mellitus. BJOG. 2016;124:1746-1752.

15. Carr SR, Slocum J, Tefft L, Haydon B, Carpenter M. Precision of office-based blood glucose meters in screening for gestational diabetes. Am J Obstet Gynecol. 1995;173:1267-1272. 
16. Balaji V, Madhuri BS, Paneerselvam A, Arthi T, Seshiah V. Comparison of venous plasma glucose and capillary whole blood glucose in the diagnosis of gestational diabetes mellitus: A community-based study. Diabetes Technol Ther. 2012;14:131-134.

17. Agarwal MM, Dhatt GS, Safraou MF. Gestational diabetes: Using a portable glucometer to simplify the approach to screening. Gynecol Obstet Invest. 2008;66:178-183.

18. Higioka AS, Rudolf-Oliveira RCM, Martinello F, Bazzo ML. Analytical performance of glucometers in a tertiary care hospital. Accred Qual Assur. 2016;21:143-149.

19. Balaji B, Manni MM, Kumar M, et al. Use of capillary blood glucose for screening for gestational diabetes mellitus in resource-constrained settings. Acta Diabetol. 2016;53:91-97.

20. Jadhav DS, Wankhede UN. Comparative study of capillary blood glucose estimation by glucometer and venous plasma glucose estimation in women undergoing the one step DIPSI test (diabetes in pregnancy study group India) for screening and diagnosis of gestational diabetes mellitus. Int J Reprod Contracept Obstet Gynecol. 2017;6:14881492.

21. Perera NJ, Molyneaux L, Constantino MI, et al. Suboptimal performance of blood glucose meters in an antenatal diabetes clinic. Diabetes Care. 2011;34:335-337.

22. Kristensen GBB, Christensen NG, Thue G, Sandberg S. Between-lot variation in external quality assessment of glucose: Clinical importance and effect on participant performance evaluation. Clin Chem. 2005;51:1632-1636.

23. Louie RF, Tang Z, Sutton DV, et al. Point-of-care glucose testing: Effexts of critical care variables, influence of reference instruments, and a modular glucose meter design. Arch Pathol Lab Med. 2000;124:257-266.

24. International Organization for Standardization: ISO 15197:2013: In Vitro Diagnostic Test Systems - Requirements for Blood-Glucose Monitoring Systems for Self-Testing in Managing Diabetes Mellitus. Published 2013.

www.iso.org/iso/catalogue_detail?csnumber=54976. Accessed June 16, 2017.

25. Essack Y, Hoffman M, Rensburg M, Van Wyk J, Meyer CS, Erasmus R. A comparison of five glucometers in South Africa. JEMDSA. 2009;14:102-105.

26. Alto WA, Meyer D, Schneid J, Bryson P, Kindig J. Assuring the accuracy of home glucose monitoring. J Am Board Fam Pract. 2002;15:1-6.

27. Kotwal N, Pandit A. Variability of capillary blood glucose monitoring measured on home glucose monitoring devices. Indian J Endocrinol Metab. 2012;16:S248-S251.

28. Ginsberg BH. Factors affecting blood glucose monitoring: Sources of errors in measurement. J Diabetes Sci Technol. 2009;3:903-913. 